\title{
Role of 18F-fluorodeoxyglucose Positron Emission Tomography/ Computed Tomography in the Evaluation of Cytologically Indeterminate Thyroid Nodules
}

\author{
Gulgun Buyukdereli, ${ }^{1,}$ Yasemin Aktar, ${ }^{1}$ Ertan Kara, ${ }^{2}$ Aysun Uguz, and Husnu Sonmez ${ }^{4}$ \\ ${ }^{1}$ Department of Nuclear Medicine, Faculty of Medicine, Cukurova University, Adana, Turkey \\ ${ }^{2}$ Department of Public Health, Faculty of Medicine, Cukurova University, Adana, Turkey \\ ${ }^{3}$ Department of Pathology, Faculty of Medicine, Cukurova University, Adana, Turkey \\ ${ }^{4}$ Department of General Surgery, Faculty of Medicine, Cukurova University, Adana, Turkey \\ ${ }^{*}$ Corresponding author: Gulgun Buyukdereli, Department of Nuclear Medicine, Faculty of Medicine, Cukurova University, Ziyapasa bulvari, Kurtulus mah, Adana, Turkey. Tel: \\ +90-5324305118, Fax:+90-3224590581, E-mail: gulgunbuyukdereli @ hotmail.com
}

Received 2014 July 3; Revised 2015 April 6; Accepted 2015 April 21.

\begin{abstract}
Background: Thyroid nodules with indeterminate fine-needle aspiration biopsy (FNAB) results remain a diagnostic dilemma, because $70-85 \%$ of these nodules have been found to be benign after thyroid surgery.

Objectives: The purpose of this study was to evaluate the usefulness of 18F-fluorodeoxyglucose (FDG) positron emission tomography/ computed tomography (PET/CT) in the preoperative diagnosis of cytologically indeterminate nodules.

Patients and Methods: Forty-six patients were included in this study. These individuals had undergone FDG PET/CTs for the preoperative evaluation of thyroid nodules with indeterminate FNAB results. The results of the preoperative PET/CT scans were compared with the postoperative pathological results and statistically analyzed.

Results: Of the 46 patients included in our study, the histopathology of the surgical specimens revealed thyroid cancer in 17 individuals $(37 \%, 17 / 46)$. The PET/CT scan showed a positive result in 27 patients. Of these, 16 patients (59.3\%) were found to have thyroid carcinomas. In addition, the PET/CT scan was considered to be negative in 19 patients, 18 (94.7\%) of whom had benign lesions. For the detection of malignant lesions, the values for the sensitivity and specificity, and the positive predictive and negative predictive values were $94 \%, 62 \%$, $59 \%$, and $95 \%$, respectively.

Conclusion: The FDG PET/CT showed a high sensitivity and a high negative predictive value for identifying malignancies in thyroid nodules with indeterminate FNAB results. Therefore, the FDG PET/CT may be a helpful tool in the clinical management of these nodules. When an FDG positive lesion is detected, further examination is recommended.
\end{abstract}

Keywords: Thyroid Nodules, FDG PET/CT, Indeterminate, Fine Needle Aspiration Biopsy

\section{Background}

Nodular thyroid disease is very common; however, most thyroid nodules, whether palpable or incidentally discovered, turn out to be benign. Only 5\% to $15 \%$ of thyroid nodules are found to be malignant (1-4). Fine needle aspiration biopsy (FNAB) is the standard method for evaluating the risk of malignancy in thyroid nodules (5-7). It is safe, easily performed, and cost effective $(8,9)$; although $15-30 \%$ of biopsied nodules exhibit indeterminate cytology (5, 10-13). The current guidelines recommend surgical resection of these nodules in order to permit sufficient pathological evaluations, but only $15-30 \%$ of them turn out to be malignant, with $70-85 \%$ being benign $(14,15)$. Therefore, most thyroid surgeries for asymptomatic thyroid nodules are actually carried out as diagnostic rather than therapeutic procedures. Because of the morbidity and potential complications associated with surgery, it is important to identify a noninvasive alternative method for the management of these nodules. Thus, the development of new diagnostic methods for indeterminate thyroid nodules is an important area of current research.

As a molecular imaging modality, 18F-fluorodeoxyglucose (FDG) positron emission tomography/computed tomography (PET/CT) can detect a wide range of malignant tissues. However, it has an unclear role in the evaluation of thyroid nodules in which the FNAB is considered to be indeterminate.

\section{Objectives}

The purpose of our study was to review the results that we had gathered, to determine the value of the FDG PET/ $\mathrm{CT}$ in the preoperative diagnosis of thyroid nodules with indeterminate FNAB results. 


\section{Patients and Methods}

Forty-six consecutive patients with thyroid nodules of indeterminate cytology, and scheduled for surgery at our institute were included in this study, prospectively, between 2009 and 2012 (Figure 1). The FDG PET/CT images were obtained from all of the patients before surgery for the evaluation of these nodules, and no treatments that could affect the results were allowed before the FDG PET/ CT imaging. All of the included patients were euthyroid, while those with uncontrolled diabetes mellitus and other known malignancies were excluded. This study was approved by the ethical board of our institute, and written informed consent was obtained from all of the patients prior to the study.

Thyroid ultrasonography was performed on all of the patients to locate those thyroid nodules requiring FNAB based on their size and appearance. Solitary or dominant thyroid nodules, which were $\geq 1 \mathrm{~cm}$ in ultrasound measurements, were selected for an FNAB. Of the nodules, 33 were solid and 13 were solid-cystic. The FNABs were performed by physicians with experience in FNAB techniques under ultrasonographic guidance, using a 23-gauge needle attached to a $10 \mathrm{~mL}$ syringe inserted into a syringe holder. The samples obtained were smeared onto glass slides and stained using the May-Grunwald Giemsa method. The FNAB reports were written according to The Bethesda system for reporting thyroid cytopathology (TBSRTC) in order to classify our cases (13). The term "indeterminate" was used to identify the "follicular neoplasm/suspicious for a follicular neoplasm" and "suspicious for malignancy" results from the TBSRTC. In all of the cases, the interval between the fine needle aspiration biopsy and FDG PET/CT was a minimum of 3 weeks.

Whole-body FDG PET/CT imaging was performed using an integrated PET/CT scanner, which consisted of a fullring high-resolution lutetium oxyorthosilicate (LSO) PET and a six-slice CT (Siemens Biograph 6; Knoxville, Tennessee, USA). The patients fasted for a minimum of 6 hours before the examination. The blood glucose levels were tested in each patient before injection, and were determined to be $<200 \mathrm{mg} / \mathrm{dL}$. Then, water-soluble iodinated contrast material diluted in $1500 \mathrm{~mL}$ of water was given to each patient orally, before scanning. A whole-body acquisition was performed 1 hour after the intravenous administration of 18F FDG, and the images were obtained from the vertex to the upper thigh region. The patients were placed on the scanner table in a supine position, and a CT topogram was acquired first, to define the axial range of the PET/CT study. Then, a CT transmission scan was acquired with a low tube current ( $130 \mathrm{kVp}, 54 \mathrm{mAs}$ ), slice thickness of $4 \mathrm{~mm}, 0.6$ seconds gantry rotation, and a collimator width of $6 \times 3 \mathrm{~mm}$. Next, PET emission scanning, with a duration of 3 minutes per bed position, was performed with an identical transverse field of view in the craniocaudal direction. The PET images were reconstructed with the CT data for attenuation correction using an iterative approach. These images were evaluated via the consensus of two experienced nuclear medicine specialists, who were blinded to the final pathological diagnoses at the time of their evaluations.

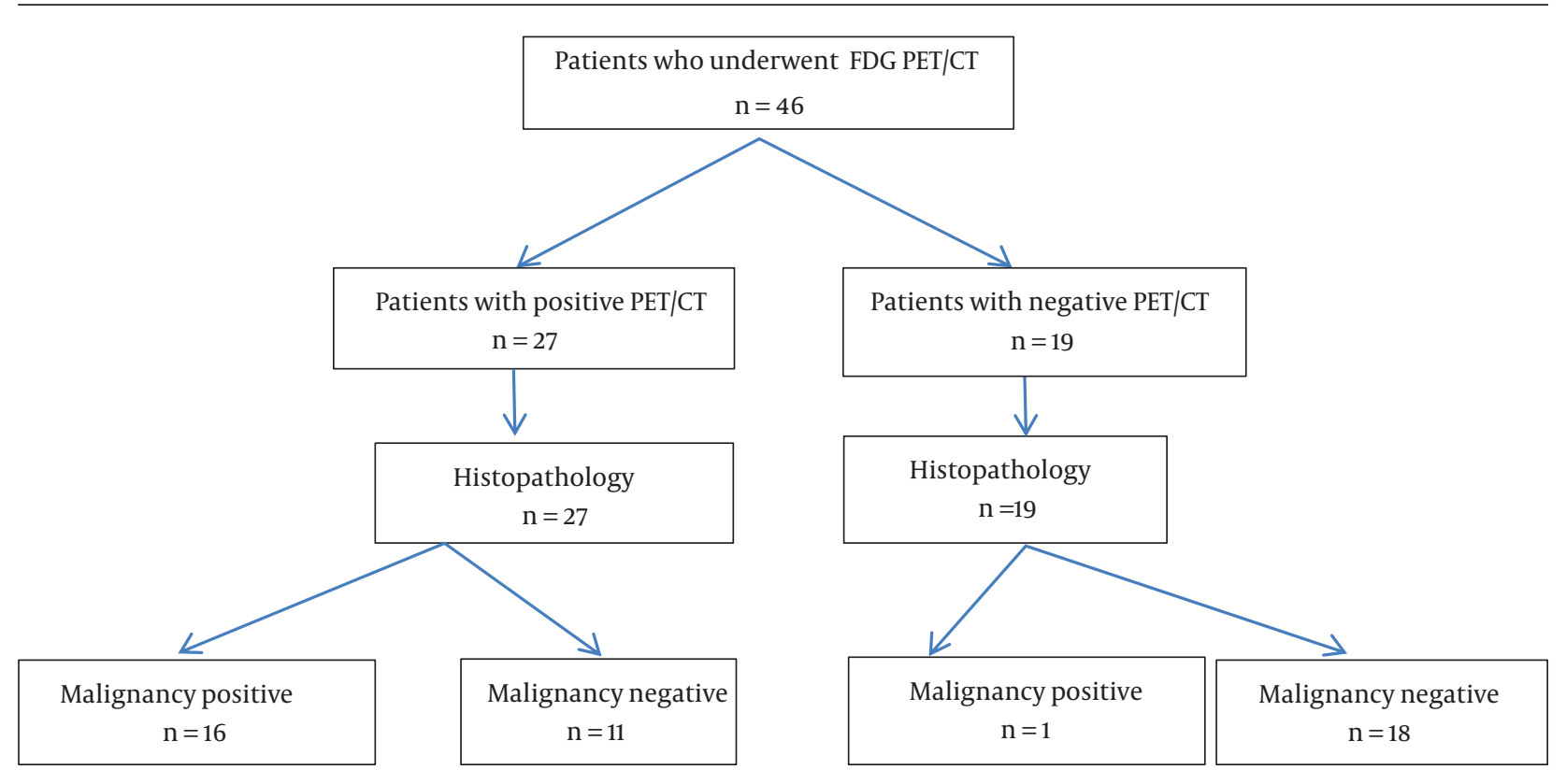

Figure 1. Flowchar of patients through the study. The FDG PET/CT was only performed on those patients scheduled for thyroidectomy on the basis of indeterminate cytology. 
The scans were classified as positive if the focal uptake was seen in the nodule under examination. The FDG uptake in the nodules was quantified by calculating the maximum standardized uptake values (SUVs); however, if the thyroid nodule was not apparent on the FDG PET/CT images, quantification was not attempted. After the FDG PET/CTs, all of the patients underwent surgical resection within a few days, and the results of the preoperative PET/ CT scans were compared with the postoperative pathological results, and statistically analyzed using IBM SPSS version 20 (IBM Corp., Released 2011. IBM SPSS Statistics for Windows, Armonk, NY). The results of the study were analyzed using the independent samples t-test, MannWhitney-U test, and Fisher's exact test. Finally, the fitness of the continuous data to normal distribution was checked by the Kolmogorov-Smirnov test, and a P value of less than 0.05 was considered to be statistically significant.

\section{Results}

Out of a total of 46 patients included in this study, 6 were men and 40 were women, with ages ranging between 18 and 74 years, and a mean age of $50.2 \pm 12.6$ years. Nineteen of the patients (41.3\%) had solitary nodules, while 27 of the patients (58.6\%) had two or more nodules. The histopathological results of the surgical specimens revealed thyroid cancer in 17 patients (37\%): 11 with papillary carcinoma $(64.7 \%, 11 / 17), 4$ with follicular carcinoma
(23.5\%, 4/17), and 2 with medullary carcinoma (11.8\%, 2/17). In the histopathological examination, the nodules were found to have a mean maximum diameter of $2.62 \pm 1.30$ cm (95\% confidence interval (CI): 2.24 - 3.00). We found that there were no statistically significant differences in the patients' age or sex, or the ultrasonographic characteristics between the malignant and benign nodules (Table 1).

The FDG PET/CT scan was found to be positive in 27 of the patients, with 16 (59.3\%) of them having thyroid carcinoma (Figures 2 and 3). The FDG PET/CT scan was considered to be negative in 19 patients, with 18 (94.7\%) patients having benign lesions and 1 (5.3\%) having thyroid carcinoma. Thus, 11 patients $(40.7 \%, 11 / 27)$ had false-positive and 1 patient $(5.2 \%, 1 / 19)$ had a false-negative FDG PET/CT scan (Table 2). The values for the sensitivity, specificity, and the positive-predictive and negative-predictive values of the FDG PET/CT in the detection of the malignant lesions were $94 \%, 62 \%, 59 \%$, and 95\%, respectively. The quantification of the FDG uptake using the SUVs could not improve this degree of accuracy. For benign lesions with focal uptake, the mean $( \pm$ SD) SUVmax was $4.46 \pm 3.71$ (range $\min =1.90 \max =14.50 \mathrm{n}=11$ ). For malignant lesions with focal uptake, the mean $( \pm S D)$ SUVmax was $5.93 \pm 3.53$ (range $\min =1,80 \max =14,00 \mathrm{n}=16$ ). However, there were no statistically significant differences found between the SUVs between the benign and malignant nodules $(\mathrm{P}=0.099)$.

\begin{tabular}{|c|c|c|c|}
\hline Patients' Characteristics & Benign & Malignant & P Value \\
\hline Age & & & 0.413 \\
\hline No. $(\%)$ & $29(63.0)$ & $17(37.0)$ & \\
\hline Mean (SD) & $49.03 \pm 13.43$ & $52.24 \pm 11.25$ & \\
\hline Size, $\mathrm{mm}$ & & & 0.381 \\
\hline No. $(\%)$ & $29(63.0)$ & $17(37.0)$ & \\
\hline Mean (SD) & $27.38 \pm 12.22$ & $24.76 \pm 14.25$ & \\
\hline SUVs & & & 0.099 \\
\hline No. $(\%)$ & $11(40.7)$ & $16(59.3)$ & \\
\hline Mean (SD) & $4.46 \pm 3.71$ & $5.93 \pm 3.53$ & \\
\hline Gender & & & 0.478 \\
\hline Female & $26(65.0)$ & $14(35.0)$ & \\
\hline Male & $3(50.0)$ & $3(50.0)$ & \\
\hline Nodularity & & & 0.220 \\
\hline Solitary & $10(52.6)$ & $9(47.4)$ & \\
\hline Multiple & $19(70.4)$ & $8(29.6)$ & \\
\hline Type of Nodule & & & 0.585 \\
\hline Solid & $20(60.6)$ & $13(39.4)$ & \\
\hline Solid-cystic & $9(69.2)$ & $4(30.8)$ & \\
\hline Echogenicity Pattern & & & 0.113 \\
\hline Hypoechogenic & $10(47.6)$ & $11(52.4)$ & \\
\hline Isoechogenic & $8(80.0)$ & $2(20.0)$ & \\
\hline Hyperechogenic & $2(100.0)$ & 0 & \\
\hline
\end{tabular}

\footnotetext{
${ }^{\mathrm{a}}$ Data are presented as mean \pm SD or No. (\%).
} 

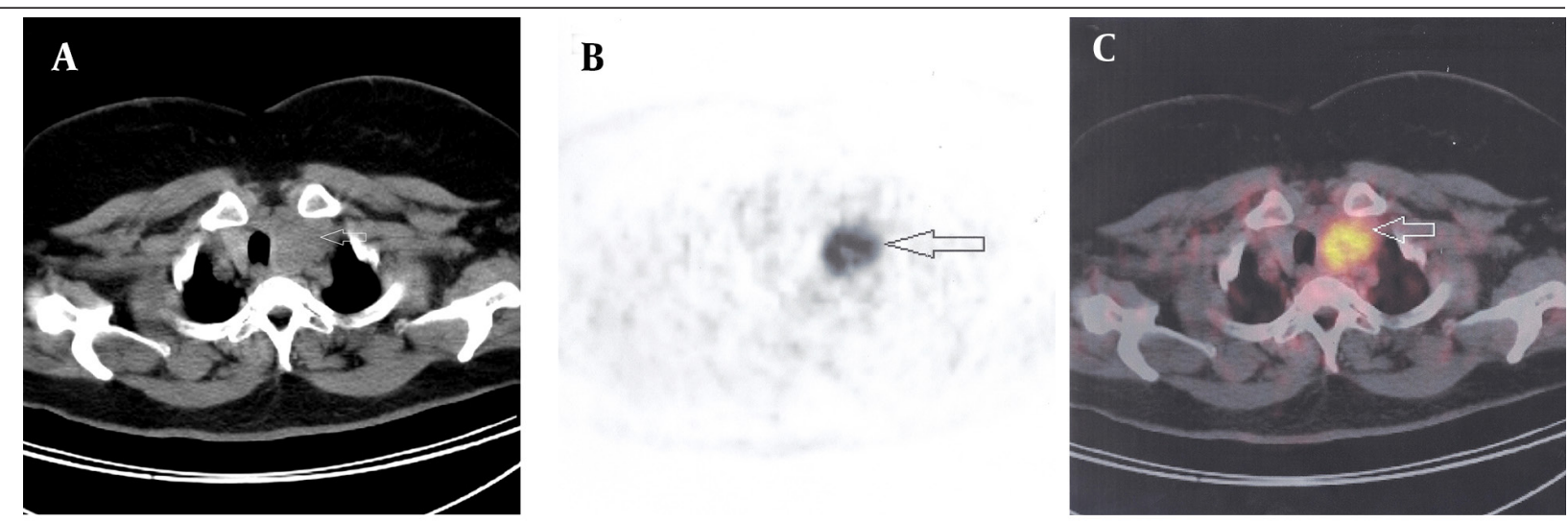

D

B

D
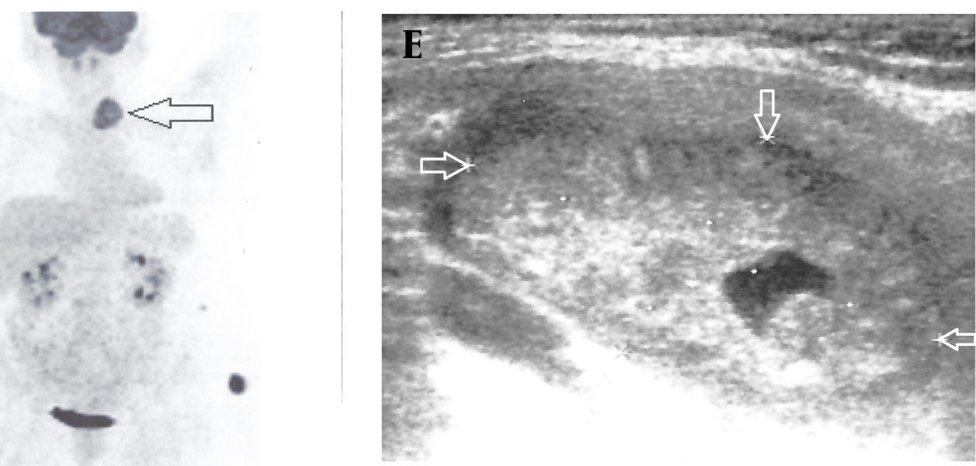

Figure 2. Images of a 61-year-old woman with a nodule in the left lobe of the thyroid (arrows). A, CT scan; B, PET scan; C, Fused PET/CT scan; D, Whole-body PET scan; E, Thyroid US image. The thyroid nodule shows increased FDG uptake (SUV=14.0) in the PET and fused PET/CT scans. The pathological assessment revealed papillary carcinoma in this patient.

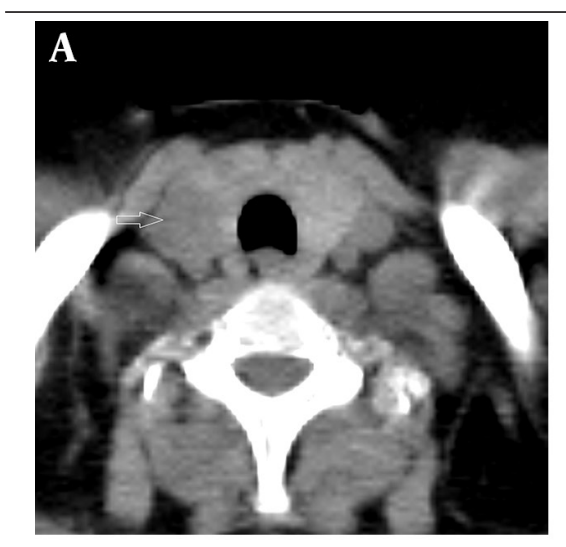

\section{B}

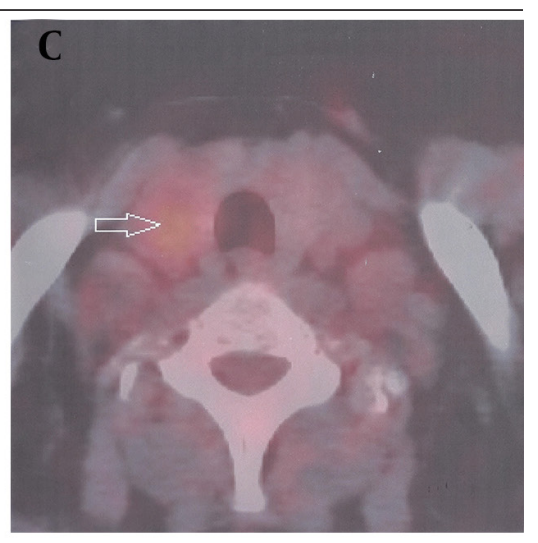

D
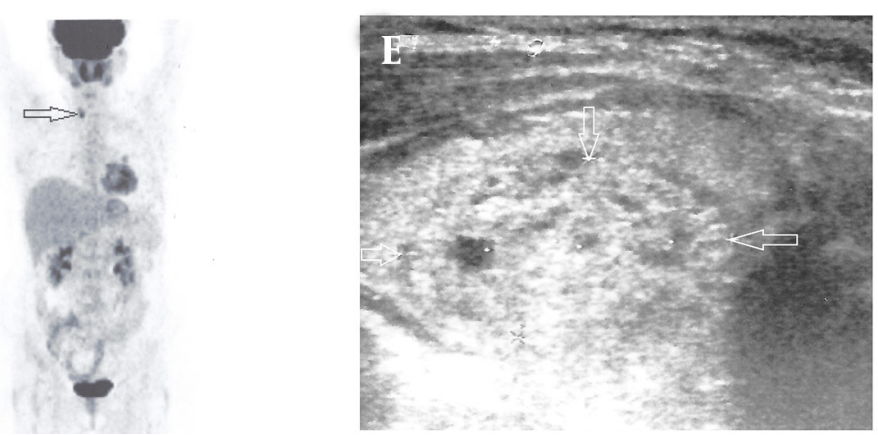

Figure 3. Images of a 47-year-old woman with a nodule in the right lobe of the thyroid (arrows). A, CT scan; B, PET scan; C, Fused PET/CT scan; D, Whole-body PET scan; E, Thyroid US image. The thyroid nodule shows increased FDG uptake (SUV=6.7) in the PET and fused PET/CT scans. The pathological assessment revealed follicular carcinoma in this patient. 
Table 2. PET Results Compared With the Malignancy Status ${ }^{\mathrm{a}}$

\begin{tabular}{lccc}
\hline & \multicolumn{2}{c}{ Malignancy } & Total \\
\cline { 2 - 3 } PET Result & Positive & Negative & \\
\hline Positive & 16 & 11 & 27 \\
Negative & 1 & 18 & 19 \\
Total & 17 & 29 & 46 \\
\hline atan
\end{tabular}

${ }^{a}$ For these lesions, sensitivity $=94 \%$, specificity $=62 \%$, positive predictive value $=59 \%$, and negative predictive value $=95 \%$.

\section{Discussion}

The FNAB plays an essential role in the evaluation of euthyroid patients with thyroid nodules, while the TBSRTC assigns a malignancy risk to each cytological category. In our study, the term "indeterminate" was used to identify "follicular neoplasm/suspicious for a follicular neoplasm" and "suspicious for malignancy" results of the TBSRTC. The distinction between follicular thyroid carcinoma and follicular adenoma cannot be made using an FNAB, and surgery is required to distinguish the benign nodules from the malignant ones. In addition, when an FNAB is suspicious for malignancy, nearly all physicians recommend surgery to exclude it. The introduction of a diagnostic test which helps to decrease the number of unnecessary surgical resections of benign nodules could have an important positive impact on patients management.

Malignant cells exhibit a number of aberrant characteristics when compared to cells in normal tissues, and these characteristics can, potentially, be used to image tumors. A PET scan with FDG relies upon the increased glucose metabolism found in a wide range of malignancies, using it to detect both primary lesions and their metastases. However, the usefulness of an FDG PET/CT in the presurgical evaluation of indeterminate thyroid nodules is conflicting, due to the contradictory results found in the literature (16-19).

In our study, $37 \%$ (17/46) of the patients demonstrated thyroid carcinomas in their final histopathological reports. The FDG PET/CT detected 16/17 malignant lesions, with a very good sensitivity of $94 \%$. Previously, Vriens et al. (17) and Wang et al. (16) meta-analyzed the usefulness of the FDG PET in evaluating thyroid nodules with indeterminate FNAB results. Sensitivity and specificity of our study similar to those found in their studies. In the study by Vriens et al. (17), it was reported that a false negative PET result was only found in those nodules with a maximum histological dimension of $\leq 1.5 \mathrm{~cm}$. In contrast, the study by Wang et al. (16) found 8 false negative cases, and 5 of them had dimensions $\geq 2 \mathrm{~cm}$. In our study, there was 1 FDG negative malignant lesion (papillary cancer), with a dimension of $2.5 \mathrm{~cm}$, and 6 FDG positive malignant lesions ( 4 papillary, 1 follicular, and 1 medullary cancer) with dimensions $\leq 1.5 \mathrm{~cm}$. Therefore, as suggested by Wang et al. (16), it is unlikely that the size of the lesion is the most important factor for explaining the false negative phenomena in our study.
In the present study, the FDG was not found to be negative in all of the benign nodules, and the FDG accumulation was detected in 11 of the 29 benign tumors. The specificity, and the positive predictive and negative predictive values were $62 \%, 59 \%$, and $95 \%$, respectively. In the SUV analysis, no significant difference was found between the benign and malignant nodules: however, there was a considerable overlap between the groups. In the previously published literature, the reports disagreed about the usefulness of the quantification of the FDG uptake, measured in terms of the SUV, for the characterization of the thyroid nodules. While some of the studies showed no significant differences between the SUVs of the benign and malignant nodules $(20,21)$, others suggested that the SUVs can be used to differentiate malignant from benign nodules(16, 22-24). However, different cutoffs for the SUVs were found in these studies and, for the most part, there was considerable overlap in their glucose metabolic activities (16, 22-24). SUVs rely on the reconstruction, acquisition, and region-of-interest parameters, and the variability found in the SUV methodology makes it difficult to compare the results obtained from different centers. Therefore, additional standardized large multicenter studies may be necessary to generalize the role of the SUV measurements in the characterization of indeterminate thyroid nodules.

For all of these reasons, regardless of the SUV measurements, we recommend further examinations on any FDG positive nodule with indeterminate cytology. According to our analysis, despite there being some false positive cases, over half of the patients could have avoided unnecessary surgeries $(62.1 \%, 18 / 29)$. However, the FDG PET/ CT is not available in some regions, and it is expensive, which could, for the time being, limit its routine use. In those patients on whom surgery cannot be performed, close regular follow-ups, including repeated FNABs and thyroid ultrasonography, should be performed. Overall, the FDG PET/CT may provide physicians with additional confidence when deciding to observe lesions, rather than to proceed immediately to surgical resection. The careful selection of those patients who could benefit most from the additional information provided by this imaging method is important, and the FDG PET/CT scan could be more useful for patients with a high risk of surgical morbidity and mortality.

In summary, our study revealed that the FDG PET/CT has high sensitivity and a negative predictive value for identifying malignancies in thyroid nodules with indeterminate cytological findings. Therefore, the FDG PET/CT may be a helpful tool in the clinical management of these nodules.

\section{Acknowledgments}

The authors would like to thank Alex Raynham for his help in checking the wording of the manuscript. 


\section{Footnotes}

Authors' Contribution:Study design, data analysis, manuscript preparation: Gulgun Buyukdereli; data collection and analysis: Yasemin Aktar; statistical analysis: Ertan Kara; pathological analysis: Aysun Uguz; clinical input: Husnu Sonmez.

Financial Disclosure:None of the authors of this study have any conflicts of interest to declare.

Funding/Support:No funding or sponsorship was received for this study.

\section{References}

1. Mazzaferri EL. Management of a solitary thyroid nodule. N Engl J Med. 1993;328(8):553-9. doi: 10.1056/NEJM199302253280807. [PubMed: 8426623]

2. Hegedus L. Clinical practice. The thyroid nodule. $N$ Engl J Med. 2004;351(17):1764-71. doi: 10.1056/NEJMcp031436. [PubMed: 15496625]

3. Sachmechi I, Miller E, Varatharajah R, Chernys A, Carroll Z, Kis$\sin \mathrm{E}$, et al. Thyroid carcinoma in single cold nodules and in cold nodules of multinodular goiters. Endocr Pract. 2000;6(1):5-7. doi: 10.4158/EP.6.1.5. [PubMed: 11419919]

4. Frates MC, Benson CB, Doubilet PM, Kunreuther E, Contreras $\mathrm{M}$, Cibas ES, et al. Prevalence and distribution of carcinoma in patients with solitary and multiple thyroid nodules on sonography. J Clin Endocrinol Metab. 2006;91(9):3411-7. doi: 10.1210/ jc.2006-0690. [PubMed:16835280]

5. American Thyroid Association Guidelines Taskforce on Thyroid N, Differentiated Thyroid C, Cooper DS, Doherty GM, Haugen BR, Kloos RT, et al. Revised American Thyroid Association management guidelines for patients with thyroid nodules and differentiated thyroid cancer. Thyroid. 2009;19(11):1167-214. doi: 10.1089/ thy.2009.0110. [PubMed: 19860577]

6. Gharib H, Papini E, Paschke R, Duick DS, Valcavi R, Hegedus L, et al. American Association of Clinical Endocrinologists, Associazione Medici Endocrinologi, and European Thyroid Association Medical guidelines for clinical practice for the diagnosis and management of thyroid nodules: executive summary of recommendations. Endocr Pract. 2010;16(3):468-75. doi: 10.4158| EP.16.3.468. [PubMed: 20551008]

7. Baloch ZW, Cibas ES, Clark DP, Layfield LJ, Ljung BM, Pitman MB, et al. The National Cancer Institute Thyroid fine needle aspiration state of the science conference: a summation. Cytojournal. 2008;5:6. doi: 10.1186/1742-6413-5-6. [PubMed:18394201]

8. Gharib H. Fine-needle aspiration biopsy of thyroid nodules: advantages, limitations, and effect. Mayo Clin Proc. 1994;69(1):44-9. [PubMed: 8271850]

9. Frates MC, Benson CB, Charboneau JW, Cibas ES, Clark OH, Coleman BG, et al. Management of thyroid nodules detected at US: Society of Radiologists in Ultrasound consensus conference statement. Radiology. 2005;237(3):794-800. doi: 10.1148/radiol.2373050220. [PubMed:16304103]

10. Gharib H, Goellner JR. Fine-needle aspiration biopsy of the thyroid: an appraisal. Ann Intern Med. 1993;118(4):282-9. [PubMed: 8420446]

11. Wang CC, Friedman L, Kennedy GC, Wang H, Kebebew E, Steward
DL, et al. A large multicenter correlation study of thyroid nodule cytopathology and histopathology. Thyroid. 2011;21(3):243-51. doi: 10.1089/thy.2010.0243. [PubMed: 21190442]

12. Grant CS, Hay ID, Gough IR, McCarthy PM, Goellner JR. Long-term follow-up of patients with benign thyroid fine-needle aspiration cytologic diagnoses. Surgery. 1989;106(6):980-5. [PubMed: 2588125]

13. Cibas ES, Ali SZ, N. C. I. Thyroid FNA State of the Science Conference. The Bethesda System For Reporting Thyroid Cytopathology. Am J Clin Pathol. 2009;132(5):658-65. doi: 10.1309/AJCPPHLWMI3JV4LA. [PubMed:19846805]

14. Gharib H, Papini E, Paschke R. Thyroid nodules: a review of current guidelines, practices, and prospects. Eur J Endocrinol. 2008;159(5):493-505. doi:10.1530/EJE-08-0135. [PubMed:18728120]

15. Bryson PC, Shores CG, Hart C, Thorne L, Patel MR, Richey L, et al. Immunohistochemical distinction of follicular thyroid adenomas and follicular carcinomas. Arch Otolaryngol Head Neck Surg. 2008;134(6):581-6. doi: 10.1001/archotol.134.6.581. [PubMed: 18559722]

16. Wang N, Zhai H, Lu Y. Is fluorine-18 fluorodeoxyglucose positron emission tomography useful for the thyroid nodules with indeterminate fine needle aspiration biopsy? A meta-analysis of the literature. J Otolaryngol Head Neck Surg. 2013;42:38. doi: 10.1186/1916-0216-42-38. [PubMed:24228840]

17. Vriens D, de Wilt JH, van der Wilt GJ, Netea-Maier RT, Oyen WJ, de Geus-Oei LF. The role of [18F]-2-fluoro-2-deoxy-d-glucose-positron emission tomography in thyroid nodules with indeterminate fine-needle aspiration biopsy: systematic review and meta-analysis of the literature. Cancer. 2011;117(20):4582-94. doi: 10.1002/ cncr.26085. [PubMed: 21432844]

18. Traugott AL, Dehdashti F, Trinkaus K, Cohen M, Fialkowski E, Quayle F, et al. Exclusion of malignancy in thyroid nodules with indeterminate fine-needle aspiration cytology after negative 18F-fluorodeoxyglucose positron emission tomography: interim analysis. World J Surg. 2010;34(6):1247-53. doi: 10.1007/s00268010-0398-3. [PubMed: 20140435]

19. Deandreis D, Al Ghuzlan A, Auperin A, Vielh P, Caillou B, Chami L, et al. Is (18)F-fluorodeoxyglucose-PET/CT useful for the presurgical characterization of thyroid nodules with indeterminate fine needle aspiration cytology? Thyroid. 2012;22(2):165-72. doi: 10.1089/thy.2011.0255. [PubMed: 22257371]

20. Joensuu H, Ahonen A, Klemi PJ.18F-fluorodeoxyglucose imaging in preoperative diagnosis of thyroid malignancy. Eur J Nucl Med. 1988;13(10):502-6. [PubMed: 3371370]

21. de Geus-Oei LF, Pieters GF, Bonenkamp JJ, Mudde AH, BleekerRovers CP, Corstens FH, et al. 18F-FDG PET reduces unnecessary hemithyroidectomies for thyroid nodules with inconclusive cytologic results. J Nucl Med.2006;47(5):770-5. [PubMed: 16644746]

22. Kresnik E, Gallowitsch HJ, Mikosch P, Stettner H, Igerc I, Gomez I, et al. Fluorine-18-fluorodeoxyglucose positron emission tomography in the preoperative assessment of thyroid nodules in an endemic goiter area. Surgery. 2003;133(3):294-9. doi: 10.1067| msy.2003.71. [PubMed: 12660642]

23. Sasaki M, Ichiya Y, Kuwabara Y, Akashi Y, Yoshida T, Fukumura T, et al. An evaluation of FDG-PET in the detection and differentiation of thyroid tumours. Nucl Med Commun. 1997;18(10):957-63. [PubMed: 9392798]

24. Mitchell JC, Grant F, Evenson AR, Parker JA, Hasselgren PO, Parangi S. Preoperative evaluation of thyroid nodules with 18FDG-PET/ CT. Surgery. 2005;138(6):1166-74. doi: 10.1016/j.surg.2005.08.031. [PubMed: 16360405] 